\title{
Asymptotic charges, large gauge transformations and inequivalence of different gauges in external current QED
}

\author{
Wojciech Dybalski ${ }^{a, 1}$ and Benedikt Wegener ${ }^{b, 2,3}$ \\ ${ }^{a}$ Zentrum Mathematik, Technische Universität München, \\ Munich, Germany \\ ${ }^{b}$ Dipartimento di Matematica, Università di Roma "Tor Vergata", \\ Rome, Italy \\ E-mail: dybalski@ma.tum.de, wegener@mat.uniroma2.it
}

ABSTRACT: In this paper we consider external current QED in the Coulomb gauge and in axial gauges for various spatial directions of the axis. For a non-zero electric charge of the current, we demonstrate that any two different gauges from this class correspond to quantum theories which are not unitarily equivalent. We show that the spacelike asymptotic flux of the electromagnetic field is the underlying superselected quantity. We also express the large gauge transformation linking two distinct axial gauges by the Wilson loop over a contour limited by the two axes. Thus the underlying physical mechanism appears to be related to the Aharonov-Bohm effect.

KEYwORDS: Gauge Symmetry, Nonperturbative Effects

ARXiv EPrint: 1907.06750

\footnotetext{
${ }^{1}$ This work was supported by the DFG within the Emmy Noether grant DY107/2-1.

${ }^{2}$ Marie Sklodowska-Curie fellow of the Istituto Nazionale di Alta Matematica.

${ }^{3}$ This project has received funding from the European Union's 2020 research and innovation programme under the Marie Sklodowska-Curie grant agreement No 713485.
} 


\section{Contents}

1 Introduction 1

2 Asymptotic charges and inequivalence of different gauges 2

3 Large gauge transformations and Wilson loops 5

4 Conclusion and outlook $\quad 10$

\section{Introduction}

In Classical Electrodynamics a change of gauge of the electromagnetic potential $A_{\mu} \mapsto$ $A_{\mu}+\partial_{\mu} \chi$ has clearly no observable effect as it does not change the electromagnetic fields $F_{\mu \nu}$. This argument does not extend to Quantum Electrodynamics, since the quantization procedures always require some gauge fixing conditions to which we refer as 'gauges'. In spite of the seemingly unlimited gauge freedom, the list of conditions used in practice is relatively short: the Lorenz gauge $\partial_{\mu} A^{\mu}=0$, the Coulomb gauge $\nabla \cdot \boldsymbol{A}=0$ and axial gauges $e_{\mu} A^{\mu}=0$ for various directions of the axis $e$ are the most common choices. Some authors have argued that the resulting theories are equivalent on the basis of partially heuristic computations $[13,21]$. In the present paper we consider external current QED (that is the second-quantized electromagnetic field coupled to an external current) in the Coulomb and axial gauges and show that actually the opposite is the case: the corresponding quantum theories are not unitarily equivalent if the current has a non-zero electric charge. Our analysis is 'ghost-free', the results are mathematically rigorous, and we exhibit a physical mechanism behind the inequivalence of different gauges.

Let us outline this mechanism briefly: it is well known that the electric charge conservation follows from the Noether theorem applied to the global U(1) symmetry. It is less well known that the Noether theorem applied to the local gauge symmetry gives conservation of the spacelike asymptotic flux of the electric field ${ }^{1}$

$$
\phi(\mathbf{n})=\lim _{r \rightarrow \infty} r^{2} \mathbf{n} \cdot \mathbf{E}(\mathbf{n} r), \text { where }|\mathbf{n}|=1,
$$

which commutes with all local observables. In an irreducible representation of the external current QED the flux $\phi$ is thus a scalar function on the unit sphere, restricted only by the Gauss Law. Any choice of this function corresponds to a different sector of the theory [3]. Not surprisingly, this function inherits the symmetry of the gauge-fixing condition used in the quantization procedure: in the Coulomb gauge it is spherically symmetric, while in the axial gauges it is only axially symmetric. Hence, the respective quantum theories are not

\footnotetext{
${ }^{1}$ The argument can be found e.g. in https://en.wikipedia.org/wiki/Infraparticle.
} 
unitarily equivalent and the same is true for axial gauges with distinct directions of the axis. However, we show that they are related by a Bogolubov transformation, which can be expressed by a Wilson loop over a contour limited by the two axes. Or, equivalently, by the flux of the magnetic field through the area surrounded by this contour. This brings to light certain similarity of our findings with the Aharonov-Bohm effect, where the phase shift of a particle traveling along two paths with the same start and end points is given by an analogous expression.

As our Bogolubov transformations change the asymptotic charge (1.1), they have a non-trivial action at infinity. In this respect they resemble the large gauge transformations considered recently in the context of the Strominger's 'infrared triangle', see e.g. $[7,8,11,14]$, which, however, are residual transformations of the Lorenz gauge. ${ }^{2}$ For transformations between the Lorenz gauge and other covariant gauges, and their automorphic action on the field algebra we refer to [10, appendix A]. For relations between the Aharonov-Bohm effect and the Gauss Law at the level of the algebra we refer to [23, 24]. An interesting discussion of the Gauss Law and the flux of the electromagnetic field in the context of axial gauges was recently given in $[2,20]$. In $[20]$ the flux is computed in axial gauges in low orders of perturbation theory, giving results similar to ours. However, the external current discussion from this reference corresponds to the Coulomb gauge in our setting. Finally, we refer to [9] and references therein for the complementary case of the second quantized Dirac field in an external electromagnetic field and its sensitivity to the choice of gauge. However, the problem of unitary inequivalence of different gauge fixing conditions is not discussed in the above references and, to our knowledge, it does not have a satisfactory treatment in the literature.

This paper is organized as follows: in section 2 we verify that the Coulomb gauge and the axial gauge are not unitarily equivalent, if the external current has a non-zero total charge. We also show the inequivalence of axial gauges with different directions of the axis. These results are obtained by computing the flux (1.1) in different gauges. In section 3 we identify the Bogolubov transformations linking different axial gauges and express them as Wilson loops. In section 4 we summarize our work, outline briefly the case of angularly smeared axial gauges and discuss future directions.

\section{Asymptotic charges and inequivalence of different gauges}

Let us first recall the standard formulas for the free transverse potential and free electromagnetic fields (see e.g. [27])

$$
\begin{aligned}
\mathbf{A}_{\perp}(t, \boldsymbol{x}) & :=\frac{1}{(2 \pi)^{3 / 2}} \sum_{\lambda= \pm} \int \frac{d^{3} \boldsymbol{k}}{\sqrt{2|\boldsymbol{k}|}} \boldsymbol{\epsilon}_{\lambda}(\boldsymbol{k})\left(e^{i|\boldsymbol{k}| t-i \boldsymbol{k} \cdot \boldsymbol{x}} a_{\lambda}^{*}(\boldsymbol{k})+e^{-i|\boldsymbol{k}| t+i \boldsymbol{k} \cdot \boldsymbol{x}} a_{\lambda}(\boldsymbol{k})\right), \\
\mathbf{E}_{\mathrm{fr}}(\boldsymbol{x}) & :=-\partial_{t} \mathbf{A}_{\perp}(t, \boldsymbol{x}), \quad \mathbf{B}_{\mathrm{fr}}(\boldsymbol{x}):=\nabla \times \mathbf{A}_{\perp}(t, \boldsymbol{x}),
\end{aligned}
$$

where $a_{\lambda}^{(*)}$ are the creation/annihilation operators of photons on the Fock space $\mathcal{F}$ and $\boldsymbol{\epsilon}_{\lambda}$ are the polarization vectors. Now we consider the electromagnetic field coupled to the

\footnotetext{
${ }^{2}$ The existence of large gauge transformations of this type can be questioned [15].
} 
time-independent external current $j=\left(j_{0}, 0\right)$, where $j_{0}$ is smooth and compactly supported. The Dirac procedure of quantization with constraints applied to this theory in the Coulomb gauge gives the familiar formulas for the electromagnetic fields (see e.g. [27])

$$
\begin{aligned}
& \mathbf{E}_{\mathrm{C}}(\boldsymbol{x}):=\mathbf{E}_{\mathrm{fr}}(\boldsymbol{x})+\frac{1}{\Delta} \nabla j_{0}(\boldsymbol{x}), \\
& \mathbf{B}_{\mathrm{C}}(\boldsymbol{x}):=\mathbf{B}_{\mathrm{fr}}(\boldsymbol{x}) .
\end{aligned}
$$

The same quantization procedure applied in the axial gauge for the axis direction $e=(0, \mathbf{e})$ gives instead (see e.g. [13])

$$
\begin{aligned}
& \mathbf{E}_{\mathbf{e}}(\boldsymbol{x}):=\mathbf{E}_{\mathrm{fr}}(\boldsymbol{x})+\frac{\mathbf{e}}{\mathbf{e} \cdot \nabla+0} j_{0}(\boldsymbol{x}), \\
& \mathbf{B}_{\mathbf{e}}(\boldsymbol{x}):=\mathbf{B}_{\mathrm{fr}}(\boldsymbol{x}) .
\end{aligned}
$$

The Dirac quantization procedure is ambiguous here, as the constraint matrix has many inverses corresponding to various regularizations of the singularity in (2.5). The choice of +0 is natural as it corresponds to a string-like localized electromagnetic potential in the axial gauge [19]. (A different derivation of (2.5), (2.6) will be given in section 3). The electromagnetic fields are operator-valued distributions and we denote by

$$
E_{\mathrm{C}}(\mathbf{f}):=\int d^{3} \boldsymbol{x} \mathbf{E}_{\mathrm{C}}(\boldsymbol{x}) \cdot \mathbf{f}(\boldsymbol{x})
$$

the smearing with an $\mathbb{R}^{3}$-valued, smooth, compactly supported function $\mathbf{f}$ (and analogously for the remaining quantities). The smeared fields are self-adjoint, unbounded operators and to avoid the discussion of domain questions we proceed to their bounded functions $\exp i \mathbf{E}_{\mathrm{C}}(\mathbf{f})$. Now we are ready to state and prove our main result:

Theorem 2.1 Suppose that $q:=\int d^{3} \boldsymbol{x} j_{0}(\boldsymbol{x}) \neq 0$. Then there is no unitary $U$ on the Fock space $\mathcal{F}$ such that

$$
U \exp i\left(\mathbf{E}_{\mathrm{C}}\left(\mathbf{f}_{\mathrm{el}}\right)+\mathbf{B}_{\mathrm{C}}\left(\mathbf{f}_{\mathrm{m}}\right)\right) U^{*}=\exp i\left(\mathbf{E}_{\mathbf{e}}\left(\mathbf{f}_{\mathrm{el}}\right)+\mathbf{B}_{\mathbf{e}}\left(\mathbf{f}_{\mathrm{m}}\right)\right)
$$

for all smearing functions $\mathbf{f}_{\mathrm{el}}, \mathbf{f}_{\mathrm{m}}$ and some fixed unit vector $\mathbf{e} \in \mathbb{R}^{3}$. (The statement remains valid if we restrict attention to smearing functions $\mathbf{f}_{\mathrm{m}} \equiv 0$ and $\mathbf{f}_{\mathrm{el}}$ supported in any fixed string $\left\{\boldsymbol{x}=|\boldsymbol{x}| \hat{\boldsymbol{x}}:|\boldsymbol{x}| \in \mathbb{R}_{+}, \hat{\boldsymbol{x}} \in \omega\right\}$, where $\omega$ is an open subset of the unit sphere).

Given two unit vectors $\mathbf{e} \neq \mathbf{e}^{\prime}$ there is no unitary $V$ on $\mathcal{F}$ s.t.

$$
V \exp i\left(\mathbf{E}_{\mathbf{e}}\left(\mathbf{f}_{\mathrm{el}}\right)+\mathbf{B}_{\mathbf{e}}\left(\mathbf{f}_{\mathrm{m}}\right)\right) V^{*}=\exp i\left(\mathbf{E}_{\mathbf{e}^{\prime}}\left(\mathbf{f}_{\mathrm{el}}\right)+\mathbf{B}_{\mathbf{e}^{\prime}}\left(\mathbf{f}_{\mathrm{m}}\right)\right)
$$

for all smearing functions $\mathbf{f}_{\mathrm{el}}, \mathbf{f}_{\mathrm{m}}$.

We remark that the assumption $q \neq 0$ is essential. Currents with $q=0$, for which different gauges are unitarily equivalent, are in abundance. This can easily be seen from the discussion in the next section.

We will prove the theorem by adapting the method of central sequence to the situation at hand (cf. $[5,18])$. Let us first show that a unitary $U$, as in $(2.8)$, cannot exist. Since 
it is clear from (2.3)-(2.6) that the problem of inequivalence is related to the electric field, we can set $\mathbf{f}_{\mathrm{m}}=0$. As $\mathbf{f}_{\text {el }}$ we choose

$$
\mathbf{f}_{\mathrm{el}, \mathbf{n}, r}(\boldsymbol{x})=\mathbf{n} \frac{1}{r} f\left(\frac{\boldsymbol{x}-\mathbf{n} r}{r}\right)
$$

where $f$ is supported in a ball around the origin of radius much smaller than one and $\mathbf{n}$ is a unit vector in $\mathbb{R}^{3}$. With this choice we have

$$
\mathbf{E}_{\mathrm{C}}\left(\mathbf{f}_{\mathrm{el}, \mathbf{n}, r}\right)=\int d^{3} \boldsymbol{x} f(\boldsymbol{x}) r^{2} \mathbf{n} \cdot \mathbf{E}_{\mathrm{C}}((\boldsymbol{x}+\mathbf{n}) r),
$$

which is a smeared version of the flux (1.1). It will be important in the later part of the proof that the following expression is independent of $r$

$$
\left\langle 0\left|e^{i \mathbf{E}_{\mathrm{fr}}\left(\mathbf{f}_{\mathrm{el}, \mathbf{n}, r}\right)}\right| 0\right\rangle=e^{-\frac{1}{4} \int d^{3} \boldsymbol{k}\left|\boldsymbol{k} \| P_{\operatorname{tr}} \mathbf{n} \tilde{f}(\boldsymbol{k})\right|^{2}}
$$

where $P_{\text {tr }}$ is the transverse projection and tilde denotes the Fourier transform.

Now we assume by contradiction that there exists a unitary $U$ as in the theorem and we compare the vacuum expectation values of the resulting equality

$$
\left\langle 0\left|U e^{i \mathbf{E}_{\mathrm{fr}}\left(\mathbf{f}_{\mathrm{el}, \mathbf{n}, r}\right)} U^{*}\right| 0\right\rangle e^{i \frac{1}{\Delta} \nabla j_{0}\left(\mathbf{f}_{\mathrm{el}, \mathbf{n}, r}\right)}=\left\langle 0\left|e^{i \mathbf{E}_{\mathrm{fr}}\left(\mathbf{f}_{\mathrm{el}, \mathbf{n}, r}\right)}\right| 0\right\rangle e^{i \frac{\mathbf{e}}{\mathbf{e} \cdot \nabla+0} j_{0}\left(\mathbf{f}_{\mathrm{el}, \mathbf{n}, r}\right)} .
$$

Since the $C^{*}$-algebra generated by the free electromagnetic fields acts irreducibly on $\mathcal{F}$, we can find, by the Kadison transitivity theorem [16, Theorem 10.2.1], a unitary $\tilde{U}$ in this algebra s.t. $U^{*}|0\rangle=\tilde{U}^{*}|0\rangle$. Then, by locality, $\lim _{r \rightarrow \infty}\left[e^{i \mathbf{E}_{\mathrm{fr}}\left(\mathbf{f}_{\mathrm{el}, \mathbf{n}, r}\right)}, \tilde{U}^{*}\right]=0$ in norm and we can write

$$
e^{i \frac{1}{\Delta} \nabla j_{0}\left(\mathbf{f}_{\mathrm{el}, \mathbf{n}, r}\right)}+o\left(r^{-1}\right)=e^{i \frac{\mathbf{e}}{\mathbf{e} \cdot \nabla+0} j_{0}\left(\mathbf{f}_{\mathrm{el}, \mathbf{n}, r}\right)}
$$

where $o\left(r^{-1}\right)$ denotes a term which tends to zero as $r \rightarrow \infty$.

Thus to conclude the proof of the first part of the theorem, we have to show that the contributions to the flux coming from the $c$-number parts in (2.3) and (2.5) are different. Concerning the Coulomb part, we have

$$
\begin{aligned}
\frac{1}{\Delta} \nabla j_{0}\left(\mathbf{f}_{\mathrm{el}, \mathbf{n}, r}\right) & =\int d^{3} \boldsymbol{x} f(\boldsymbol{x}) \int d^{3} \mathbf{y} \frac{r^{2} \mathbf{n} \cdot(\mathbf{n} r+\boldsymbol{x} r-\mathbf{y})}{4 \pi|\mathbf{n} r+\boldsymbol{x} r-\mathbf{y}|^{3}} j_{0}(\mathbf{y}) \\
& \rightarrow \underset{r \rightarrow \infty}{\rightarrow} \int d^{3} \boldsymbol{x} f(\boldsymbol{x}) \frac{q \mathbf{n} \cdot(\mathbf{n}+\boldsymbol{x})}{4 \pi|\mathbf{n}+\boldsymbol{x}|^{3}}
\end{aligned}
$$

We note in passing that in the limit of no smearing $(f(\boldsymbol{x}) \rightarrow \delta(\boldsymbol{x}))$ we obtain a spherically symmetric distribution $\frac{q}{4 \pi}$ which manifestly respects the Gauss Law. As for the axial part, 
we set $\mathbf{n}_{\boldsymbol{x}}:=\mathbf{n}+\boldsymbol{x}$ and compute

$$
\begin{aligned}
\frac{\mathbf{e}}{\mathbf{e} \cdot \nabla+0} j_{0}\left(\mathbf{f}_{\mathrm{e}, \mathbf{n}, r}\right) & =\left.\int d^{3} \boldsymbol{x} f(\boldsymbol{x}) \lim _{\epsilon \rightarrow 0} \frac{r^{2} \mathbf{n} \cdot \mathbf{e}}{\mathbf{e} \cdot \nabla_{\mathbf{y}}+\epsilon} j_{0}(\mathbf{y})\right|_{\mathbf{y}=r \mathbf{n}_{\boldsymbol{x}}} \\
& =\left.\int d^{3} \boldsymbol{x} f(\boldsymbol{x})\left(r^{2} \mathbf{n} \cdot \mathbf{e}\right) \lim _{\epsilon \rightarrow 0} \int_{0}^{\infty} d s e^{-\left(\mathbf{e} \cdot \nabla_{\mathbf{y}}+\epsilon\right) s} j_{0}(\mathbf{y})\right|_{\mathbf{y}=r \mathbf{n}_{\boldsymbol{x}}} \\
& =\int d^{3} \boldsymbol{x} f(\boldsymbol{x})\left(r^{2} \mathbf{n} \cdot \mathbf{e}\right) \int_{0}^{\infty} d s j_{0}\left(r \mathbf{n}_{\boldsymbol{x}}-s \mathbf{e}\right) \\
& =\int d^{3} \boldsymbol{x} f(\boldsymbol{x})\left(r^{2} \mathbf{n} \cdot \mathbf{e}\right) \int_{0}^{\infty} d s j_{0}\left(\left(r\left(\mathbf{n}_{\boldsymbol{x}} \cdot \mathbf{e}\right)-s\right) \mathbf{e}+r P_{\mathbf{e}}^{\perp} \mathbf{n}_{\boldsymbol{x}}\right) \\
& =\int d^{3} \boldsymbol{x} f(\boldsymbol{x})\left(r^{2} \mathbf{n} \cdot \mathbf{e}\right) \int_{-r\left(\mathbf{n}_{\boldsymbol{x}} \cdot \mathbf{e}\right)}^{\infty} d s j_{0}\left(-s \mathbf{e}+r P_{\mathbf{e}}^{\perp} \mathbf{n}_{\boldsymbol{x}}\right),
\end{aligned}
$$

where $P_{\mathbf{e}}^{\perp}=1-|\mathbf{e}\rangle\langle\mathbf{e}|$. Suppose first that $\mathbf{n}$ is not parallel to $\mathbf{e}$. Then, if the support of $f$ is in a sufficiently small neighbourhood of zero, we have $P_{\mathbf{e}}^{\perp} \mathbf{n}_{\boldsymbol{x}} \neq 0$ and $j_{0}\left(-s \mathbf{e}+r P_{\mathbf{e}}^{\perp} \mathbf{n}_{\boldsymbol{x}}\right)=0$ for $r$ sufficiently large and all $s$. Next, suppose that $\mathbf{n}=-\mathbf{e}$. Then, again for a sufficiently small support of $f$, we have that $\left(\mathbf{n}_{\boldsymbol{x}} \cdot \mathbf{e}\right)<0$ and the expression vanishes for $r$ sufficiently large due to the shrinking of the region of $s$-integration. Finally we consider the case $\mathbf{n}=\mathbf{e}$. Assuming that $\mathbf{e}$ is in the direction of the third axis of the coordinate frame, we can rewrite expression (2.16) as follows

$$
\begin{aligned}
(2.16) & =\int d^{3} \boldsymbol{x} f(\boldsymbol{x}) r^{2} \int_{0}^{\infty} d s j_{0}\left(r x_{1}, r x_{2},(r-s)+r x_{3}\right) \\
& =\int d^{3} \boldsymbol{x} f\left(x_{1}, x_{2}, x_{3}\right) r^{2} \int_{-\infty}^{r\left(1+x_{3}\right)} d s^{\prime} j_{0}\left(r x_{1}, r x_{2}, s^{\prime}\right) \\
& =\int d y_{1} d y_{2} d x_{3} f\left(y_{1} / r, y_{2} / r, x_{3}\right) \int_{-\infty}^{r\left(1+x_{3}\right)} d s^{\prime} j_{0}\left(y_{1}, y_{2}, s^{\prime}\right) \\
& \rightarrow \underset{r}{\rightarrow} q \int d x_{3} f\left(0,0, x_{3}\right) .
\end{aligned}
$$

Thus, summing up, we have

$$
\frac{\mathbf{e}}{\mathbf{e} \cdot \nabla+0} j_{0}\left(\mathbf{f}_{\mathrm{el}, \mathbf{n}, r}\right)= \begin{cases}q \int d s f(s \mathbf{e}) & \text { for } \mathbf{n}=\mathbf{e}, \\ 0 & \text { for } \mathbf{n} \neq \mathbf{e} .\end{cases}
$$

We note as an aside that the first line in (2.19) is singular in the limit $f(\boldsymbol{x}) \rightarrow \delta(\boldsymbol{x})$, thus there is no conflict with the Gauss Law here. By comparing the second line of (2.19) with (2.15) we easily obtain a contradiction in (2.14) in the limit $r \rightarrow \infty$. The second part of the theorem is proven analogously, making use of the first line in (2.19).

\section{Large gauge transformations and Wilson loops}

In this section we establish relations between different gauges which are less restrictive than unitary equivalence and thus not in conflict with Theorem 2.1. We first introduce a general class of gauge transformations of the external current QED, which is initially in 
the Coulomb gauge. Then we identify a transformation mapping the Coulomb gauge into the axial gauge theory for a given direction of the axis.

We recall that the Coulomb gauge electromagnetic potential and the Hamiltonian have the form

$$
\begin{array}{lc}
A_{0, \mathrm{C}}(t, \boldsymbol{x}):=-\frac{1}{\Delta} j_{0}(\boldsymbol{x}), & \mathbf{A}_{\mathrm{C}}(t, \boldsymbol{x}):=\mathbf{A}_{\perp}(t, \boldsymbol{x}), \\
H_{\mathrm{C}}:=H_{\mathrm{fr}}+\frac{1}{2} \int d^{3} \boldsymbol{x} A_{0, \mathrm{C}}(\boldsymbol{x}) j_{0}(\boldsymbol{x}), & \text { where } H_{\mathrm{fr}}:=\sum_{\lambda= \pm} \int d^{3} \boldsymbol{k}|\boldsymbol{k}| a_{\lambda}^{*}(\boldsymbol{k}) a_{\lambda}(\boldsymbol{k}) .
\end{array}
$$

The resulting electromagnetic fields are given by (2.3), (2.4). While the minimal coupling is not manifest from the above formulas (note that $H_{\mathrm{C}}$ depends quadratically on $j_{0}$ ), its remnant is the following relation

$$
\frac{\delta H_{\mathrm{C}}}{\delta j_{0}(\boldsymbol{x})}=A_{0, \mathrm{C}}(\boldsymbol{x})
$$

To change the gauge we introduce a family of operator-valued distributions $\boldsymbol{x} \mapsto \chi_{\epsilon}(\boldsymbol{x})$ s.t. $\left[\chi_{\epsilon}(\boldsymbol{x}), \chi_{\epsilon}\left(\boldsymbol{x}^{\prime}\right)\right]=0$ for $\boldsymbol{x}, \boldsymbol{x}^{\prime} \in \mathbb{R}^{3}$. They depend on a regularization parameter $\epsilon>0$, whose role will become clear in the example below, and which eventually will tend to zero. For non-zero $\epsilon$, and after smearing with real-valued test functions, these distributions are assumed to yield self-adjoint operators. Thus we can define the unitaries

$$
W_{\epsilon}:=e^{-i \chi_{\epsilon}\left(j_{0}\right)}
$$

Setting $\chi_{\epsilon}(t, \boldsymbol{x}):=e^{i H_{\mathrm{C}} t} \chi_{\epsilon}(\boldsymbol{x}) e^{-i H_{\mathrm{C}} t}$, we define a new potential as follows

$$
\begin{aligned}
A_{\epsilon, 0}(t, \boldsymbol{x}) & :=W_{\epsilon}\left(A_{0, \mathrm{C}}(t, \boldsymbol{x})+\partial_{t} \chi_{\epsilon}(t, \boldsymbol{x})\right) W_{\epsilon}^{*}, \\
\mathbf{A}_{\epsilon}(t, \boldsymbol{x}) & :=W_{\epsilon}\left(\mathbf{A}_{\mathrm{C}}(t, \boldsymbol{x})-\nabla \chi_{\epsilon}(t, \boldsymbol{x})\right) W_{\epsilon}^{*} .
\end{aligned}
$$

Clearly, the resulting electromagnetic fields

$$
\begin{aligned}
& \mathbf{E}_{\epsilon}(t, \boldsymbol{x})=-\partial_{t} \mathbf{A}_{\epsilon}(t, \boldsymbol{x})-\nabla A_{0, \epsilon}(t, \boldsymbol{x})=W_{\epsilon} \mathbf{E}_{\mathrm{C}}(t, \boldsymbol{x}) W_{\epsilon}^{*}, \\
& \mathbf{B}_{\epsilon}(t, \boldsymbol{x})=\nabla \times \mathbf{A}_{\epsilon}(t, \boldsymbol{x})=W_{\epsilon} \mathbf{B}_{\mathrm{C}}(t, \boldsymbol{x}) W_{\epsilon}^{*},
\end{aligned}
$$

satisfy the Maxwell equations with the same current $j$. Their time-evolution is governed by the Hamiltonian

$$
H_{\epsilon}:=W_{\epsilon} H_{\mathrm{C}} W_{\epsilon}^{*} .
$$

The presence of the transformation $W_{\epsilon}(\ldots) W_{\epsilon}^{*}$ in (3.5), (3.6) calls for a justification. We remark that the Maxwell equations are insensitive to this transformation in our external current situation. ${ }^{3}$ The same is true for the Dirac brackets, as they only fix the algebraic relations between the potentials and the fields, and not the representation of the resulting

\footnotetext{
${ }^{3}$ For Pauli-Fierz type models with a dynamical electron the quantum Maxwell equations depend on the electromagnetic potential via the electron's velocity (see e.g. [25, formula (13.51)]). Then the forminvariance of the Maxwell equations necessitates a transformation analogous to $W_{\epsilon}(\ldots) W_{\epsilon}^{*}$ in the definition of gauge transformations.
} 
algebra. However, global quantities, like the Hamiltonian, may be sensitive to the choice of the representation. It is therefore not a surprise that the role of the transformation $W_{\epsilon}(\ldots) W_{\epsilon}^{*}$ is to preserve the minimal coupling property (3.3), as can be seen from the following computation:

$$
\frac{\delta H_{\epsilon}}{\delta j_{0}(\boldsymbol{x})}=e^{-i \chi_{\epsilon}\left(j_{0}\right)} \frac{\delta H_{\mathrm{C}}}{\delta j_{0}(\boldsymbol{x})} e^{i \chi_{\epsilon}\left(j_{0}\right)}+e^{-i \chi_{\epsilon}\left(j_{0}\right)}\left[-i \chi_{\epsilon}(\boldsymbol{x}), H_{\mathrm{C}}\right] e^{i \chi_{\epsilon}\left(j_{0}\right)}=A_{\epsilon, 0}(\boldsymbol{x}) .
$$

Let us now focus on the transformation from the Coulomb to axial gauge. By imposing the axial gauge condition $\mathbf{e} \cdot \mathbf{A}_{\epsilon}(\boldsymbol{x}) \rightarrow 0$ as $\epsilon \rightarrow 0$ we read off from (3.6)

$$
\chi_{\mathbf{e}, \epsilon}(\boldsymbol{x})=\frac{1}{\mathbf{e} \cdot \nabla-\epsilon} \mathbf{e} \cdot \mathbf{A}_{\mathrm{C}}(\boldsymbol{x}) .
$$

We remark that the choice of the $-\epsilon$ prescription will prove consistent with the choice made in (2.5). The corresponding family of unitaries (3.4), similar to transformations considered in [13], has the form

$$
W_{\mathbf{e}, \epsilon}:=\exp \left(i \int_{0}^{\infty} d s e^{-\epsilon s}\left(\mathbf{e} \cdot \mathbf{A}_{\mathrm{C}}\right)\left(j_{0}\right)(s \mathbf{e})\right),
$$

where we used $(\mathbf{e} \cdot \nabla-\epsilon)^{-1}=-\int_{0}^{\infty} d s e^{(\mathbf{e} \cdot \nabla-\epsilon) s}$. As we show in Lemma 3.1 below, the resulting electromagnetic fields (3.7), (3.8) coincide with the axial gauge electromagnetic fields (2.5), (2.6) from the previous section. This lemma should be compared with the first part of Theorem 2.1. The key point here is that $W_{\mathbf{e}, \epsilon}$ does not converge to a well-defined unitary in the limit $\epsilon \rightarrow 0$ if $q \neq 0$. (For $q=0$ such a limiting unitary may exist, and the statement of Theorem 2.1 may not be valid). Nevertheless, $\lim _{\epsilon \rightarrow 0} W_{\mathbf{e}, \epsilon}(\cdot) W_{\mathbf{e}, \epsilon}^{*}$ does exist and defines a Bogolubov transformation or, in other words, an automorphism of the $C^{*}$-algebra of the free electromagnetic fields.

Lemma 3.1 For a fixed unit vector $\mathbf{e} \in \mathbb{R}^{3}$ and all smearing functions $\mathbf{f}_{\mathrm{el}}, \mathbf{f}_{\mathrm{m}}$ we have

$$
\lim _{\epsilon \rightarrow 0} W_{\mathbf{e}, \epsilon} \exp i\left(\mathbf{E}_{\mathrm{C}}\left(\mathbf{f}_{\mathrm{el}}\right)+\mathbf{B}_{\mathrm{C}}\left(\mathbf{f}_{\mathrm{m}}\right)\right) W_{\mathbf{e}, \epsilon}^{*}=\exp i\left(\mathbf{E}_{\mathbf{e}}\left(\mathbf{f}_{\mathrm{el}}\right)+\mathbf{B}_{\mathbf{e}}\left(\mathbf{f}_{\mathrm{m}}\right)\right) .
$$

This lemma is a consequence of the canonical commutation relations

$$
\left[A_{\perp, i}(\boldsymbol{x}),-E_{\mathrm{fr}, j}\left(\boldsymbol{x}^{\prime}\right)\right]=i \delta_{i, j}^{\perp}\left(\boldsymbol{x}-\boldsymbol{x}^{\prime}\right):=i(2 \pi)^{-3} \int d^{3} \boldsymbol{k} e^{-i \boldsymbol{k} \cdot\left(\boldsymbol{x}-\boldsymbol{x}^{\prime}\right)}\left(\delta_{i, j}-\hat{k}_{i} \hat{k}_{j}\right)
$$

and the following computation

$$
\begin{aligned}
W_{\mathbf{e}, \epsilon} E_{\mathrm{fr}, j}(\boldsymbol{x}) W_{\mathbf{e}, \epsilon}^{*}-E_{\mathrm{fr}, j}(\boldsymbol{x})= & {\left[i \int_{0}^{\infty} d s e^{-\epsilon s}\left(\mathbf{e} \cdot \mathbf{A}_{\perp}\right)\left(j_{0}\right)(s \mathbf{e}), E_{\mathrm{fr}, j}(\boldsymbol{x})\right] } \\
= & -i \int_{0}^{\infty} d s \int d^{3} \boldsymbol{x}^{\prime} e^{-\epsilon s} i e_{i} \delta_{i, j}^{\perp}\left(s \mathbf{e}+\boldsymbol{x}^{\prime}-\boldsymbol{x}\right) j_{0}\left(\boldsymbol{x}^{\prime}\right) \\
= & \frac{1}{(2 \pi)^{3 / 2}} \int_{0}^{\infty} d s \int d^{3} \boldsymbol{k} e^{-i(\boldsymbol{k} \cdot \mathbf{e}-i \epsilon) s+i \boldsymbol{k} \cdot \boldsymbol{x}} e_{i}\left(\delta_{i, j}-\hat{k}_{i} \hat{k}_{j}\right) \tilde{j}_{0}(\boldsymbol{k}) \\
= & \frac{1}{(2 \pi)^{3 / 2}} \int d^{3} \boldsymbol{k} e^{i \boldsymbol{k} \cdot \boldsymbol{x}} \frac{1}{i(\boldsymbol{k} \cdot \mathbf{e}-i \epsilon)} e_{j} \tilde{j_{0}}(\boldsymbol{k}) \\
& -\frac{1}{(2 \pi)^{3 / 2}} \int d^{3} \boldsymbol{k} e^{i \boldsymbol{k} \cdot \boldsymbol{x}} \frac{1}{i(\boldsymbol{k} \cdot \mathbf{e}-i \epsilon)} \frac{(\mathbf{e} \cdot \boldsymbol{k})}{|\boldsymbol{k}|^{2}} k_{j} \tilde{j}_{0}(\boldsymbol{k})
\end{aligned}
$$




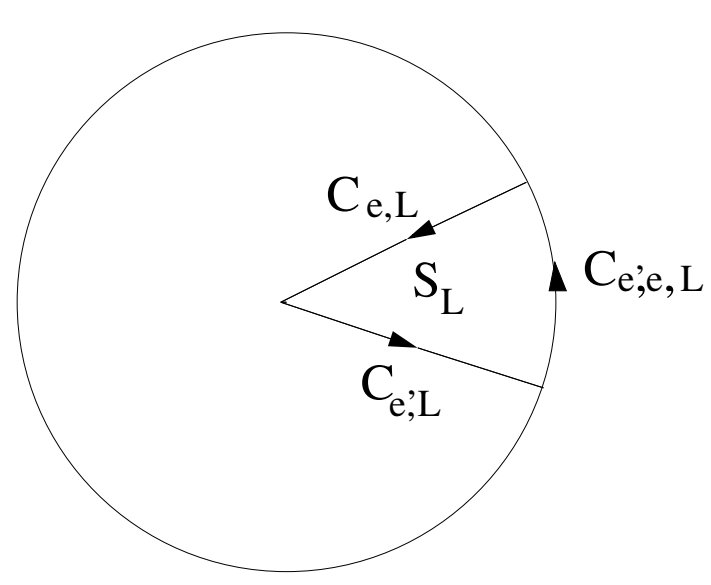

Figure 1. Contour of integration in (3.22).

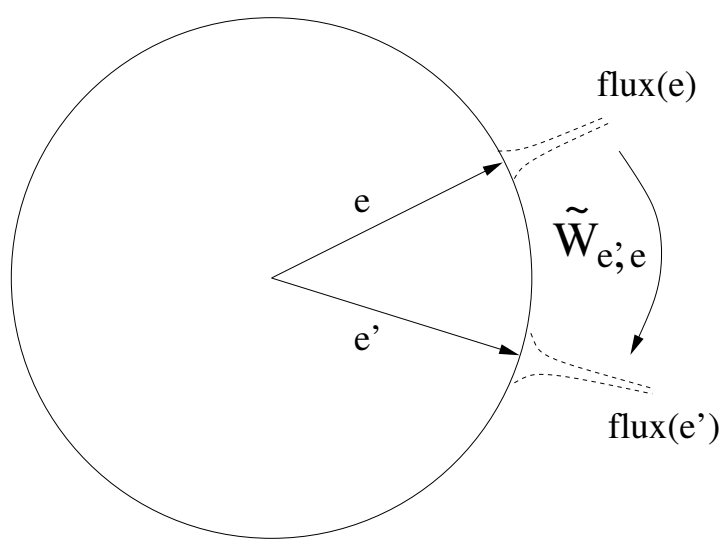

Figure 2. $\widetilde{W}_{\mathbf{e}^{\prime}, \mathbf{e}}$ as a string-local flux-carrying field.

We recall formulas (2.3) and (2.5), and note that

$$
\begin{aligned}
& (3.15)=\frac{e_{j}}{\mathbf{e} \cdot \nabla+\epsilon} j_{0}(\boldsymbol{x}), \\
& (3.16)=\frac{1}{(2 \pi)^{3 / 2}} \int d^{3} \boldsymbol{k} e^{i \boldsymbol{k} \cdot \boldsymbol{x}} \frac{1}{|\boldsymbol{k}|^{2}} i k_{j} \tilde{j_{0}}(\boldsymbol{k})+o(\epsilon) \underset{\epsilon \rightarrow 0}{\rightarrow}-\frac{\nabla_{j} j_{0}(\boldsymbol{x})}{\Delta},
\end{aligned}
$$

where $o(\epsilon)$ denotes a term which tends to zero as $\epsilon \rightarrow 0$. Thus we conclude the proof of Lemma 3.1, since $W_{\mathbf{e}, \epsilon}$ commutes with the magnetic fields.

From (3.13) we can easily read off the large gauge transformation linking two axial gauges with axes $\mathbf{e} \neq \mathbf{e}^{\prime}$. The relation

$$
\lim _{\epsilon \rightarrow 0} W_{\mathbf{e}^{\prime}, \epsilon} W_{\mathbf{e}, \epsilon}^{*} \exp i\left(\mathbf{E}_{\mathbf{e}}\left(\mathbf{f}_{\mathrm{el}}\right)+\mathbf{B}_{\mathbf{e}}\left(\mathbf{f}_{\mathrm{m}}\right)\right) W_{\mathbf{e}, \epsilon} W_{\mathbf{e}^{\prime}, \epsilon}^{*}=\exp i\left(\mathbf{E}_{\mathbf{e}^{\prime}}\left(\mathbf{f}_{\mathrm{el}}\right)+\mathbf{B}_{\mathbf{e}^{\prime}}\left(\mathbf{f}_{\mathrm{m}}\right)\right)
$$

should be compared with the second part of Theorem 2.1. It turns out that this transformation has interesting geometric properties. To bring them to light, we change the regularization method. That is, we define an auxiliary family of transformations

$$
W_{\mathbf{e}, L}:=\exp \left(i \int_{0}^{L} d s\left(\mathbf{e} \cdot \mathbf{A}_{\mathrm{C}}\right)\left(j_{0}\right)(s \mathbf{e})\right)
$$

and check by a straightforward computation that (3.19) remains true if the operators $W_{\mathbf{e}, \epsilon}, W_{\mathbf{e}^{\prime}, \epsilon}$ are replaced with $W_{\mathbf{e}, L}, W_{\mathbf{e}^{\prime}, L}$ and the limit $L \rightarrow \infty$ is taken. Clearly, we can write

$$
W_{\mathbf{e}^{\prime}, \mathbf{e}, L}:=W_{\mathbf{e}^{\prime}, L} W_{\mathbf{e}, L}^{*}=\exp \left(i \int_{0}^{L} d s^{\prime}\left(\mathbf{e}^{\prime} \cdot \mathbf{A}_{\mathrm{C}}\right)\left(j_{0}\right)\left(s^{\prime} \mathbf{e}^{\prime}\right)+i \int_{-L}^{0} d s\left((-\mathbf{e}) \cdot \mathbf{A}_{\mathrm{C}}\right)\left(j_{0}\right)(s(-\mathbf{e}))\right) .
$$

Let us denote the two regions of integration above by $C_{\mathbf{e}, L}, C_{\mathbf{e}^{\prime}, L}$. Aiming at a Wilson loop, we close $C_{\mathbf{e}, L} \cup C_{\mathbf{e}^{\prime}, L}$ with a contour $C_{\mathbf{e}^{\prime}, \mathbf{e}, L}$ depicted in figure 1. We set $\partial S_{L}:=$ $C_{\mathbf{e}, L} \cup C_{\mathbf{e}^{\prime}, L} \cup C_{\mathbf{e}^{\prime}, \mathbf{e}, L}$ and define a new transformation

$$
\widetilde{W}_{\mathbf{e}^{\prime}, \mathbf{e}, L}:=\exp \left(i \int_{\partial S_{L}} \mathbf{A}_{\mathrm{C}}\left(j_{0}\right)(\mathbf{r}) \cdot d \mathbf{r}\right)=\exp \left(i \int_{S_{L}} \mathbf{B}_{\mathrm{C}}\left(j_{0}\right)(\mathbf{r}) \cdot d \boldsymbol{S}\right),
$$


where in the second step we used the Stokes law and $S_{L}$ is the surface enclosed by $\partial S_{L}$. Since $\mathbf{B}_{\mathrm{C}}$ is a local field, we see that the resulting Bogolubov transformation acts trivially on all the observables spacelike separated w.r.t. the region $\bigcup_{L>0} S_{L}+\operatorname{supp} j_{0}$. Due to the following theorem, closing the contour of integration has no effect in the limit $L \rightarrow \infty$. Thus the operation of changing the axial gauge from $\mathbf{e}$ to $\mathbf{e}^{\prime}$ is localised in the string defined by the two axes (see figure 2). In the light of formula (2.19), this operation has the physical meaning of carrying the flux of the electric field (1.1) from one axis direction to another. This observation may be relevant for a development of superselection theory for local gauge invariance in the spirit of the Doplicher-Haag-Roberts analysis [12, chapter IV].

Theorem 3.2 For any fixed unit vectors $\mathbf{e} \neq \mathbf{e}^{\prime}$ and all smearing functions $\mathbf{f}_{\mathrm{el}}, \mathbf{f}_{\mathrm{m}}$ we have

$$
\lim _{L \rightarrow \infty} \widetilde{W}_{\mathbf{e}^{\prime}, \mathbf{e}, L} \exp i\left(\mathbf{E}_{\mathbf{e}}\left(\mathbf{f}_{\mathrm{el}}\right)+\mathbf{B}_{\mathbf{e}}\left(\mathbf{f}_{\mathrm{m}}\right)\right) \widetilde{W}_{\mathbf{e}^{\prime}, \mathbf{e}, L}^{*}=\exp i\left(\mathbf{E}_{\mathbf{e}^{\prime}}\left(\mathbf{f}_{\mathrm{el}}\right)+\mathbf{B}_{\mathbf{e}^{\prime}}\left(\mathbf{f}_{\mathrm{m}}\right)\right) .
$$

Clearly, it suffices to show that the contribution to $\lim _{L \rightarrow \infty} \widetilde{W}_{\mathbf{e}^{\prime}, \mathbf{e}, L}(\cdot) \widetilde{W}_{\mathbf{e}^{\prime}, \mathbf{e}, L}^{*}$ coming from the contour $C_{\mathbf{e}^{\prime}, \mathbf{e}, L}$ acts as the identity on the electromagnetic fields. As it is trivially true for the magnetic fields, it suffices to show that

$$
\lim _{L \rightarrow \infty} \exp \left(i \int_{C_{\mathbf{e}^{\prime}, \mathbf{e}, L}} \mathbf{A}_{\mathrm{C}}\left(j_{0}\right)(\mathbf{r}) \cdot d \mathbf{r}\right) e^{i \mathbf{E}_{\mathrm{fr}}\left(\mathbf{f}_{\mathrm{el}}\right)} \exp \left(-i \int_{C_{\mathbf{e}^{\prime}, \mathbf{e}, L}} \mathbf{A}_{\mathrm{C}}\left(j_{0}\right)(\mathbf{r}) \cdot d \mathbf{r}\right)=e^{i \mathbf{E}_{\mathrm{fr}}\left(\mathbf{f}_{\mathrm{el}}\right)}
$$

For this purpose we compute, using the canonical commutation relations (3.14),

$$
\begin{aligned}
& {\left[\int_{C_{\mathbf{e}, \mathbf{e}^{\prime}, L}} \mathbf{A}_{\perp}\left(j_{0}\right)(\mathbf{r}) \cdot d \mathbf{r},-E_{\mathrm{fr}, j}\left(\mathbf{f}_{\mathrm{el}}\right)\right]} \\
& \quad=\int d^{3} \boldsymbol{x} \int d^{3} \mathbf{y} j_{0}(\boldsymbol{x})\left(\mathbf{f}_{\mathrm{el}}\right)_{j}(\mathbf{y}) \int_{C_{\mathrm{e}^{\prime}, \mathbf{e}, L}}(d \mathbf{r})_{i}\left[A_{\perp, i}(\boldsymbol{x}+\mathbf{r}),-E_{\mathrm{fr}, j}(\mathbf{y})\right] \\
& \quad=i \int d^{3} \boldsymbol{x} \int d^{3} \mathbf{y} j_{0}(\boldsymbol{x})\left(\mathbf{f}_{\mathrm{el}}\right)_{j}(\mathbf{y}) \int_{C_{\mathbf{e}^{\prime}, \mathbf{e}, L}}(d \mathbf{r})_{i}(2 \pi)^{-3} \int d^{3} \boldsymbol{k} e^{-i \boldsymbol{k} \cdot(\boldsymbol{x}+\mathbf{r}-\mathbf{y})\left(\delta_{i, j}-\hat{k}_{i} \hat{k}_{j}\right)} \\
& \quad=i \int_{C_{\mathbf{e}^{\prime}, \mathbf{e}, L}}(d \mathbf{r})_{i} \int d^{3} \boldsymbol{k} e^{-i \boldsymbol{k} \cdot \mathbf{r}}\left(\delta_{i, j}-\hat{k}_{i} \hat{k}_{j}\right) \tilde{j}_{0}(\boldsymbol{k})\left(\tilde{\mathbf{f}}_{\mathrm{el}}\right)_{j}(-\boldsymbol{k}) \\
& =i \int_{C_{\mathbf{e}^{\prime}, \mathbf{e}, L}}(d \mathbf{r})_{i} \delta_{i, j} \int d^{3} \boldsymbol{x} j_{0}(\boldsymbol{x})\left(\mathbf{f}_{\mathrm{el}}\right)_{j}(\boldsymbol{x}+\mathbf{r}) \\
& \quad+i \int_{C_{\mathbf{e}^{\prime}, \mathbf{e}, L}}(d \mathbf{r})_{i} \int d^{3} \boldsymbol{x} j_{0}(\boldsymbol{x}) \int d^{3} \mathbf{y} \frac{1}{4 \pi|\mathbf{y}+\mathbf{r}|} \partial_{i} \partial_{j}\left(\mathbf{f}_{\mathrm{el}}\right)_{j}(\boldsymbol{x}-\mathbf{y}) .
\end{aligned}
$$

We note that (3.25) vanishes for sufficiently large $L$ since $j_{0}$ and $\mathbf{f}_{\mathrm{el}}$ are compactly supported. As for (3.26), we write in polar coordinates $\mathbf{r}=L \hat{\mathbf{r}}$ and assume that the contour is in the plane $\left\{x_{3}=0\right\}$. Then $d \mathbf{r}=L \hat{\varphi} d \varphi$ and we have

$$
(3.26)=i \int_{0}^{\varphi_{0}} d \varphi \hat{\boldsymbol{\varphi}}_{i} \int d^{3} \boldsymbol{x} j_{0}(\boldsymbol{x}) \int d^{3} \mathbf{y} \frac{L}{4 \pi|\mathbf{y}+L \hat{\mathbf{r}}|} \partial_{i} \partial_{j}\left(\mathbf{f}_{\mathrm{el}}\right)_{j}(\boldsymbol{x}-\mathbf{y}) .
$$

We take the limit $L \rightarrow \infty$ and then the integral w.r.t. $\mathbf{y}$ gives zero. 


\section{Conclusion and outlook}

In this paper we demonstrated the unitary inequivalence of different gauge-fixing conditions in the presence of a non-zero electric charge. This was achieved by exhibiting an asymptotic charge which distinguished different gauges. Furthermore, we presented a general formalism for computing large gauge transformations linking different gauges. We showed that the transformation pertaining to the change of the axis direction of the axial gauge is given by a Wilson loop over the region confined by the two axes. Although our analysis was restricted to the external current situation, we believe that the main conclusions remain valid in a larger generality. In particular, it is very plausible that the flux of the electric field (1.1) retains the symmetry of the gauge fixing condition also in the presence of dynamical charged particles.

It is well known that the axial gauge is very singular. In our case, the Hamiltonian (3.9) exists in the limit $\epsilon \rightarrow 0$ only as a quadratic form and a presence of a self-adjoint Hamiltonian can be excluded using criteria from [22]. Similarly, $A_{0, \epsilon}$ diverges in the limit $\epsilon \rightarrow 0$. These problems can be resolved by considering smeared axial gauges in the spirit of [19]. This amounts to replacing (3.11) with

$$
\chi_{g, \epsilon}(\boldsymbol{x})=\int d \Omega(\mathbf{e}) g(\mathbf{e}) \frac{1}{\mathbf{e} \cdot \nabla-\epsilon} \mathbf{e} \cdot \mathbf{A}_{\mathrm{C}}(\boldsymbol{x}),
$$

where $d \Omega$ is the spherical measure and $g$ is a smooth, positive function, normalized to one on the sphere $S^{2}$. For this choice of $\chi$ the Hamiltonian and the electromagnetic potential are well defined. It is not straightforward to relate the smeared axial gauge to the method of Dirac brackets, but it is possible [26]. The discussion from the present paper can be adapted to the gauges of the form (4.1). In particular, for $g \equiv C_{g} \neq 0$ on a subset $O_{g} \subset S^{2}$ and $g \equiv 0$ outside of some larger set $\tilde{O}_{g}$ we have the following counterpart of formula (2.19)

$$
\lim _{r \rightarrow \infty} \int d \Omega(\mathbf{e}) g(\mathbf{e}) \frac{\mathbf{e}}{\mathbf{e} \cdot \nabla+0} j_{0}\left(\mathbf{f}_{\mathrm{el}, \mathbf{n}, r}\right)= \begin{cases}C_{g} \int d^{3} \boldsymbol{x} f(\boldsymbol{x}) \frac{q \mathbf{n} \cdot(\mathbf{n}+\boldsymbol{x})}{4 \pi|\mathbf{n}+\boldsymbol{x}|^{3}} & \text { for } \mathbf{n} \in O_{g}, \\ 0 & \text { for } \mathbf{n} \notin \tilde{O}_{g} .\end{cases}
$$

It is obtained by similar steps as (2.19) and making use of the simple fact that for $g \equiv 1 /(4 \pi)$ formula (4.1) reproduces the Coulomb gauge. We note as an aside that the limit $r \rightarrow \infty$ in (4.2) cannot be interchanged with the integral over $d \Omega$, as then the result would be zero by (2.19). Exploiting (4.2), one easily shows the inequivalence of different smeared axial gauges, for suitable choices of smearing functions, in analogy with Theorem 2.1. Furthermore, for a generic non-constant $g$ the Hamiltonian $H_{g}$ in the smeared axial gauge does not have a ground state (cf. [1, formula (32)]). In this case there is clearly no unitary $U$ s.t. $U H_{g} U^{*}=H_{\mathrm{C}}$, since $H_{\mathrm{C}}$ has a ground state. Summing up, the case of smeared axial gauges demonstrates that Theorem 2.1 is not just a manifestation of the known pathologies of the sharp axial gauge.

It is an interesting question for future research how to reconcile the results of the present paper with the generally expected gauge independence. One approach is to immerse the system in a highly fluctuating but low-energetic background radiation ('infravacuum') and try to restore the unitary equivalence of different gauges. Concrete examples of such infravacua can be found in $[5,17,18]$. Another approach is to restrict attention to observables 
localised in a fixed future lightcone, so that the fluxes (1.1) cannot be measured. Then one can try to prove the unitary equivalence on the resulting subalgebra, building on ideas from $[4,6]$. We hope to come back to these questions in future investigations.

\section{Acknowledgments}

W.D. would like to thank Yoh Tanimoto and Kasia Rejzner for numerous interesting discussions in the course of this work. Thanks are also due to Daniela Cadamuro, Jens Mund and Bert Schroer for comments on the manuscript. The hospitality of the Perimeter Institute, Waterloo, and the Hausdorff Research Institute for Mathematics, Bonn, is gratefully acknowledged. Thanks are also due to the organizers of the conferences 'Infrared Problems in QED and Quantum Gravity', Perimeter Institute, Waterloo, and 'Foundational and Structural Aspects of Gauge Theories', MITP Mainz, which stimulated this research.

Open Access. This article is distributed under the terms of the Creative Commons Attribution License (CC-BY 4.0), which permits any use, distribution and reproduction in any medium, provided the original author(s) and source are credited.

\section{References}

[1] H.-J. Borchers, R. Haag and B. Schroer, The vacuum state in quantum field theory, Nuovo Cim. 29 (1963) 148.

[2] D. Buchholz, F. Ciolli, G. Ruzzi and E. Vasselli, On string-localized potentials and gauge fields, arXiv:1904.10055 [INSPIRE].

[3] D. Buchholz, The physical state space of quantum electrodynamics, Commun. Math. Phys. 85 (1982) 49 [INSPIRE].

[4] D. Buchholz and J.E. Roberts, New light on infrared problems: sectors, statistics, symmetries and spectrum, Commun. Math. Phys. 330 (2014) 935 [arXiv:1304.2794] [INSPIRE].

[5] D. Cadamuro and W. Dybalski, Relative normalizers of automorphism groups, infravacua and the problem of velocity superselection in QED, arXiv:1807.07919 [INSPIRE].

[6] D. Cadamuro and W. Dybalski, Curing velocity superselection in non-relativistic QED by restriction to a lightcone, arXiv:1902.09478 [INSPIRE].

[7] M. Campiglia and A. Laddha, Asymptotic symmetries of QED and Weinberg's soft photon theorem, JHEP 07 (2015) 115 [arXiv: 1505. 05346] [INSPIRE].

[8] M. Campiglia and R. Eyheralde, Asymptotic U(1) charges at spatial infinity, JHEP 11 (2017) 168 [arXiv: 1703. 07884] [InSPIRE].

[9] D.-A. Deckert, D. Dür, F. Merkl and M. Schottenloher, Time evolution of the external field problem in QED, J. Math. Phys. 51 (2010) 122301 [arXiv:0906. 0046] [INSPIRE].

[10] F. Finster and A. Strohmaier, Gupta-Bleuler quantization of the Maxwell field in globally hyperbolic space-times, Ann. H. Poincaré 16 (2015) 1837 [Erratum ibid. 19 (2018) 323] [arXiv: 1307.1632] [INSPIRE].

[11] B. Gabai and A. Sever, Large gauge symmetries and asymptotic states in QED, JHEP 12 (2016) 095 [arXiv: 1607.08599] [INSPIRE]. 
[12] R. Haag, Local quantum physics, second edition, Springer-Verlag, Berlin, Germany (1996).

[13] K. Haller and E. Lim-Lombridas, Quantum gauge equivalence in QED, Found. Phys. 24 (1994) 217 [hep-th/9306008] [INSPIRE].

[14] T. He, P. Mitra, A.P. Porfyriadis and A. Strominger, New symmetries of massless QED, JHEP 10 (2014) 112 [arXiv:1407.3789] [INSPIRE].

[15] A. Herdegen, Asymptotic structure of electrodynamics revisited, Lett. Math. Phys. 107 (2017) 1439 [arXiv: 1604.04170] [INSPIRE].

[16] R.V. Kadison and J.R. Ringrose, Fundamentals of the theory of operator algebras: advanced theory, Academic Press, U.S.A. (1986).

[17] K. Kraus, L. Polley and G. Reents, Models for infrared dynamics. I. Classical currents, Ann. Inst. H. Poincaré 26 (1977) 109.

[18] W. Kunhardt, On infravacua and the localization of sectors, J. Math. Phys. 39 (1998) 6353 [math-ph/9806003] [INSPIRE].

[19] J. Mund, B. Schroer and J. Yngvason, String-localized quantum fields and modular localization, Commun. Math. Phys. 268 (2006) 621 [math-ph/0511042] [INSPIRE].

[20] J. Mund, K.-H. Rehren and B. Schroer, Gauss' law and string-localized quantum field theory, arXiv: 1906.09596 [INSPIRE].

[21] Y. Nakawaki, A. Tanaka and K. Ozaki, Verification of equivalence of the axial gauge to the Coulomb gauge in QED by embedding in the indefinite metric Hilbert space, Prog. Theor. Phys. 91 (1994) 579 [INSPIRE].

[22] G. Roepstorff, Coherent photon states and spectral condition, Commun. Math. Phys. 19 (1970) 301 [INSPIRE].

[23] K. Sanders, C. Dappiaggi and T.-P. Hack, Electromagnetism, local covariance, the Aharonov-Bohm effect and Gauss' law, Commun. Math. Phys. 328 (2014) 625 [arXiv: 1211.6420] [INSPIRE].

[24] B. Schroer, The role of positivity and causality in interactions involving higher spin, Nucl. Phys. B 941 (2019) 91 [arXiv:1712.02346] [INSPIRE].

[25] H. Spohn, Dynamics of charged particles and their radiation fields, Cambridge University Press, Cambridge, U.K. (2004) [INSPIRE].

[26] B. Wegener, The problem of inequivalence of different gauges in external current QED, MSc thesis, Technische Universität München/Ludwig-Maximilians-Universität München, Munich, Germany (2018).

[27] S. Weinberg, The quantum theory of fields: volume 1, foundations, Cambridge University Press, Cambridge, U.K. (1995) [INSPIRE]. 